\title{
CONVERGENCE OF A CLASS OF CUBIC INTERPOLATORY SPLINES
}

\author{
A. ChATTERJEe AND H. P. DIKSHIT
}

\begin{abstract}
The interpolation problem of matching a cubic spline at one intermediate point and cubic spline with multiple knots at two intermediate points between the successive knots are studied when the interpolatory points are not necessarily equispaced.
\end{abstract}

1. Introduction. The interpolation problems of matching a cubic spline at one intermediate point and cubic spline with multiple knots (see [1, p. 125]) at two intermediate points between the successive knots have been studied in [3]. The problems of matching the integral means of splines between successive knots with the same means of a given function for quadratic and cubic splines have been respectively settled by Sharma and Tzimbalario [4] and Dikshit [2]. It may be observed that the results [3, Theorem 1] and [2, Theorem 1] do not cover the interpolation problem when the points of interpolation are not equispaced. The object of the present paper is to study the problems of one point interpolation by cubic splines and two point interpolation by cubic splines with multiple knots when the interpolatory points are not necessarily equispaced.

2. Existence and uniqueness (equidistant knots). Let us consider the partition $\boldsymbol{P}$ of $[0,1]$ given by $P: 0=x_{0}<x_{1}<\cdots<x_{n}=1$ such that $x_{i}-x_{i-1}=p$ for $i=$ $1,2, \ldots, n$. For a positive integer $m$, we define by $\Pi_{m}$ the class of polynomials of degree $m$ or less. We say that $s(x)$ is a $p p$ function of degree $m$ over $P$ if the restriction $s_{i}(x)$ of $s(x)$ over $\left[x_{i-1}, x_{i}\right]$ is in $\Pi_{m}$ for $i=1,2, \ldots, n$. The class $S(3, P)$ of cubic splines is the set of $p p$ functions of degree three which are in the class $C^{2}[0,1]$.

Considering the points

$$
y_{i}(a)=y_{i}=x_{i-1}+a_{i} p, \quad i=1, \ldots, n,
$$

with $0<a_{i}<1$, we propose the following:

PROBlem A. Under what restrictions on $a_{i}$ does there exist a unique spline in $S(3, P)$ satisfying the interpolatory condition

$$
f\left(y_{i}\right)=s\left(y_{i}\right), \quad i=1, \ldots, n,
$$

where the $f\left(y_{i}\right)$ 's are given functional values?

Received by the editors January 28, 1980.

1980 Mathematics Subject Classification. Primary 41A05, 65D05; Secondry 41A15, 65D07.

Key words and phrases. Cubic spline, interpolation, nonequispaced interpolatory points, existence and uniqueness, convergence. 
In order to investigate Problem $A$, we observe that since $s(x) \in S(3, P), s^{\prime \prime}(x)$ is linear; therefore, for the interval $\left[x_{i-1}, x_{i}\right]$, we may write

$$
6 p s(x)=M_{i}\left(x-x_{i-1}\right)^{3}+M_{i-1}\left(x_{i}-x\right)^{3}+3 A_{i}\left(x-y_{i}\right)+B_{i}
$$

where $M_{i}=s^{\prime \prime}\left(x_{i}\right)$ and $A_{i}, B_{i}$ are appropriate constants which have to be determined.

We are now set to answer Problem A with the following

THEOREM 1. Let $f$ be 1-periodic. Then there exists a unique 1-periodic spline $s(x)$ in the class $S(3, P)$ satisfying the interpolatory condition (2.1) if for all $i$ either $0<a_{i}<$ $1 / 3$ or $2 / 3<a_{i}<1$.

It may be observed that in the interpolation problem considered in [3], $a_{i}$ is a constant so that the points of interpolation are required to be equispaced.

Proof of Theorem 1. Using the requirement that $s(x)$ is in the class $C^{2}[0,1]$, we get

$$
\begin{aligned}
A_{i+1}-A_{i} & =2 M_{i} p^{2}, \\
B_{i+1}-B_{i} & =3 a_{i+1} p A_{i+1}+3\left(1-a_{i}\right) p A_{i} .
\end{aligned}
$$

Thus, in view of the interpolatory condition (2.1), we get

$$
\begin{aligned}
a_{i+2}^{3} b_{i} M_{i+2}+ & {\left[\left(-a_{i+1}^{3}+\alpha_{i+2}^{3}+6 a_{i+2}\right) b_{i}-a_{i+1}^{3} b_{i+1}\right] M_{i+1} } \\
& +\left[\left(6 \alpha_{i}-\alpha_{i+1}^{3}+a_{i}^{3}\right) b_{i+1}-\alpha_{i+1}^{3} b_{i}\right] M_{i}+\alpha_{i}^{3} b_{i+1} M_{i-1} \\
= & 6 p^{-2}\left[b_{i+1} \Delta f\left(y_{i}\right)-b_{i} \Delta f\left(y_{i+1}\right)\right]
\end{aligned}
$$

where $\alpha_{i}=1-a_{i}, b_{i}=1-a_{i}+a_{i+1}$ and $\Delta f\left(y_{i}\right)=f\left(y_{i}\right)-f\left(y_{i+1}\right)$.

It is clear that the coefficients of $M_{i+2}, M_{i-1}$ are nonnegative. We next observe that the coefficients of $M_{i+1}$ and $M_{i}$ are positive in both the cases: (i) $0<a_{i}<1 / 3$ and (ii) $2 / 3<a_{i}<1$. Considering (i), we see that $\alpha_{i+2}^{3}-a_{i+1}^{3}>(7 / 27)$ whereas $a_{i+1}^{3}<1 / 27$; therefore, the coefficient of $M_{i+1}$ is not less than $\left(7 b_{i}-b_{i+1}\right) / 27$ which is positive since $7 b_{i}>b_{i+1}$. We also see that if (ii) holds, then $6 a_{i+2}-a_{i+1}^{3}$ $>3$ whereas $a_{i+1}^{3}<1$; therefore, the coefficient of $M_{i+1}$ is not less than $3 b_{i}-b_{i+1}$ $>0$. By a parallel reasoning we may show that the coefficient of $M_{i}$ is also positive in each of the two cases.

In (2.5), the excess of the coefficient of $M_{i}$ over the sum of the coefficients of $M_{i+2}, M_{i+1}$ and $M_{i-1}$ is

$$
\begin{aligned}
c_{i}= & {\left[1-3 a_{i}^{2}+2 a_{i}^{3}-3 a_{i+1}^{2}+2 a_{i+1}^{3}\right] b_{i+1} } \\
& +\left[1-3 a_{i+1}^{2}+2 a_{i+1}^{3}-3 a_{i+2}^{2}\right] b_{i} .
\end{aligned}
$$

If we now assume that, for all $i, 0<a_{i}<1 / 3$, then $c_{i}$ is clearly seen to be positive since $b_{i}, b_{i+1}$ and their coefficients are all positive. Thus, the coefficient matrix of (2.5) is diagonally dominant whenever $0<a_{i}<1 / 3$.

For the other case we observe that the excess of the coefficient of $M_{i+1}$ over the sum of the coefficients of $M_{i+2}, M_{i}$ and $M_{i-1}$ in (2.5) is

$$
\begin{aligned}
d_{i}= & {\left[-1+3 a_{i+1}^{2}-2 a_{i+1}^{3}+3 a_{i+2}^{2}-2 a_{i+2}^{3}\right] b_{i} } \\
& +\left[-3+6 a_{i}-3 a_{i}^{2}+3 a_{i+1}^{2}-2 a_{i+1}^{3}\right] b_{i+1}
\end{aligned}
$$


But, if we assume that, for all $i, 2 / 3<a_{i}<1$, then the coefficients of $b_{i}$ and $b_{i+1}$ in (2.7) are positive monotonic nondecreasing functions of $a_{i+1}, a_{i+2}$ and $a_{i}, a_{i+1}$ respectively whenever $2 / 3<a_{i}, a_{i+1}, a_{i+2}<1$. Thus $d_{i}>0$ and the coefficient matrix of (2.5) is diagonally dominant. This completes the proof of Theorem 1.

3. Error bounds (one point interpolation). In this section we obtain error bounds for the spline interpolant of Theorem 1. For simplicity, we assume throughout this section that for all $i$

$$
a_{i}-a_{i+1}=k
$$

where $k$ is a constant. In what follows, we shall use the notation that, for the function $f(x), f_{i}=f\left(x_{i}\right)$. We shall prove the following.

TheOREM 2. Let $f$ be a 1-periodic function in the class $C^{2}[0,1]$ and $s(x)$ be its spline interpolant in $S(3, P)$ of Theorem 1 . Then for $r=0,1,2$,

$$
\left\|(s-f)^{(r)}(x)\right\|<n^{r-2}\left(K+K_{r}|k|\right) w\left(f^{\prime \prime} ; 1 / n\right)
$$

where $w$ denotes the modulus of continuity and $K, K_{r}$ are positive constants.

Proof of Theorem 2. Writing $M_{i}-f_{i}^{\prime \prime}=e_{i}$, we have from (2.5) after some simplifications,

$$
\begin{aligned}
a_{i+2}^{3} e_{i+2} & +\left(-2 a_{i+1}^{3}+\alpha_{i+2}^{3}+6 a_{i+2}\right) e_{i+1} \\
+ & \left(6 \alpha_{i}-2 \alpha_{i+1}^{3}+a_{i}^{3}\right) e_{i}+\alpha_{i}^{3} e_{i-1}=F_{i}
\end{aligned}
$$

where

$$
\begin{aligned}
F_{i}= & 3(1-k)^{2}\left(f^{\prime \prime}\left(u_{i}\right)+f^{\prime \prime}\left(u_{i+1}\right)\right)-a_{i+2}^{3} f_{i+2}^{\prime \prime}-\alpha_{i}^{3} f_{i-1}^{\prime \prime} \\
& -\left(-2 a_{i+1}^{3}+\alpha_{i+2}^{3}+6 a_{i+2}\right) f_{i+1}^{\prime \prime}-\left(6 \alpha_{i}-2 \alpha_{i+1}^{3}+a_{i}^{3}\right) f_{i}^{\prime \prime}
\end{aligned}
$$

and $u_{i}, u_{i+1}$ are some points in $\left(y_{i}, y_{i+1}\right)$ and $\left(y_{i+1}, y_{i+2}\right)$ respectively. Writing $d\left(f_{i}^{\prime \prime}\right)=f^{\prime \prime}\left(u_{i}\right)-f_{i}^{\prime \prime}$, we have

$$
\begin{aligned}
F_{i}= & a_{i+2}^{3} \Delta f_{i+1}^{\prime \prime}+\left(-2 a_{i+1}^{3}+3 a_{i+1}^{2}+3 a_{i+1}-2-6 a_{i+1} k+3 k\right) \Delta f_{i}^{\prime \prime} \\
& +\left(a_{i}^{3}-3 a_{i+1}^{2}+3 a_{i+1}-1-6 a_{i+1} k+3 k-3 k^{2}\right) \Delta f_{i-1}^{\prime \prime} \\
& +\left(3-6 k+3 k^{2}\right)\left[d\left(f_{i}^{\prime \prime}\right)+d\left(f_{i+1}^{\prime \prime}\right)\right]
\end{aligned}
$$

so that

$$
\left\|F_{i}\right\|<\left(N_{1}^{\prime}+N_{2}^{\prime}|k|\right) w\left(f^{\prime \prime} ; 1 / n\right)
$$

where $N_{1}^{\prime}, N_{2}^{\prime}$ are appropriate positive constants.

We now proceed to obtain the error bound for the case when $0<a_{i}<1 / 3$ for all $i$ and observe that similar bounds hold for the other case. In view of the assumption that $a_{i}-a_{i+1}=k$, we see from (2.6) that the row max norm of the coefficient matrix in (3.2) is not less than

$$
2-3 a_{i}^{2}+2 a_{i}^{3}-6 a_{i+1}^{2}+4 a_{i+1}^{3}-3 a_{i+2}^{2}
$$

which in its turn is not less than $8 / 9$, since (3.5) is a positive monotonic nonincreasing function of $a_{i}, a_{i+1}, a_{i+2}$ whenever $0<a_{i}<1 / 3$ for all $i$. Thus, the row $\max$ norm of the inverse of the coefficient matrix in (3.2) does not exceed $9 / 8$, so that, 
from (3.2) and (3.4) we have

$$
\left\|e_{i}\right\|<\left(N_{1}^{\prime \prime}+K_{2}|k|\right) w\left(f^{\prime \prime} ; 1 / n\right)
$$

where $N_{1}^{\prime \prime}=9 N_{1}^{\prime} / 8$ and $K_{2}=9 N_{2}^{\prime} / 8$.

Since $s^{\prime \prime}(x)$ is linear, it follows from (3.6) that

$$
\left\|s^{\prime \prime}(x)-f^{\prime \prime}(x)\right\|<\left(K+K_{2}|k|\right) w\left(f^{\prime \prime} ; 1 / n\right)
$$

where $K=N_{1}^{\prime \prime}+1$.

It may be observed that $s^{\prime}(x)-f^{\prime}(x)$ vanishes for at least one point $v_{i}$ in $\left(y_{i}, y_{i+1}\right)$ by Rolle's Theorem. Thus, from (3.7) we get

$$
\left\|s^{\prime}(x)-f^{\prime}(x)\right\|<n^{-1}\left(K+K_{1}|k|\right) w\left(f^{\prime \prime} ; 1 / n\right)
$$

when we observe that, for $x \in\left[y_{i}, y_{i+1}\right],\left|x-v_{i}\right|<n^{-1}(1+|k|)$. A further integration yields

$$
\|s(x)-f(x)\|<\frac{1}{n^{2}}\left(K+K_{0}|k|\right) w\left(f^{\prime \prime} ; \frac{1}{n}\right) .
$$

This completes the proof of Theorem 2.

REMARK 3.1. Taking $k=0$ in (3.1), so that $a_{i+1}=a_{i}$ for all $i$, it may be easily seen from (3.3) that $\left\|F_{i}\right\|<(88 / 9) w\left(f^{\prime \prime} ; 1 / n\right)$. Thus, it follows that

$$
\left\|\left(s^{\prime \prime}-f^{\prime \prime}\right)(x)\right\|<12 w\left(f^{\prime \prime} ; 1 / n\right) \text {. }
$$

This case of Theorem 2 corresponds to the result proved in [3, Theorem 1], where we have 15 in place of 12 in the inequality (3.10).

4. Cubic spline with multiple knots. Even those $p p$ functions which do not satisfy the maximum (nontrivial) smoothness requirement have been discovered to be quite interesting (see [1, p. 125]). The set of 1-periodic pp functions of degree 3 which are in $C^{1}[0,1]$ define the class $S^{*}(3, P)$ of periodic cubic splines with multiple knots. In $\S \S 4$ and 5 we shall study the following problem of interpolation by $p p$ functions of the class $S^{*}(3, P)$.

PROBLem B. Under what restrictions on $a_{i}$ does there exist a unique spline $s(x)$ in the class $S^{*}(3, P)$ satisfying the interpolatory conditions:

$$
f\left(y_{i}\right)=s\left(y_{i}\right) \text { and } f\left(t_{i}\right)=s\left(t_{i}\right), \quad i=1, \ldots, n,
$$

where $y_{i}=x_{i-1}+a_{i} p, t_{i}=x_{i-1}+3 p / 4$ and the $f\left(y_{i}\right)$ 's and $f\left(t_{i}\right)$ 's are given functional values?

Observing that any $s(x)$ in $S^{*}(3, P)$ is also in $C^{1}[0,1]$ and writing $m_{i}=s^{\prime}\left(x_{i}\right)$, we have

$$
\begin{aligned}
p^{3} s(x)= & -(1 / 3) m_{i} p\left[p+5\left(x_{i}-x\right)\right]\left(x-x_{i-1}\right)^{2}-(1 / 3) m_{i-1} p\left(x_{i}-x\right)^{3} \\
& +\left(D_{i+1}-D_{i}\right)\left(p+2\left(x_{i}-x\right)\right)\left(x-x_{i-1}\right)^{2}+D_{i} p^{3}
\end{aligned}
$$

For convenience, we set $J_{i}=\left(9+12 a_{i}-16 a_{i}^{2}\right)^{-1}$ and $d=27 p /(32)^{2}$. Now using the interpolatory condition $s\left(t_{i}\right)=f\left(t_{i}\right)$, we have

$$
f\left(t_{i}\right)=-16 d m_{i}-(16 / 81) d m_{i-1}+32 p^{-1} d\left(D_{i+1}-D_{i}\right)+D_{i} .
$$


Eliminating $D_{i+1}-D_{i}$ between (4.2) and (4.3) and using the other interpolatory condition in (4.1), we have

$$
\begin{aligned}
J_{i}^{-1} D_{i}= & (9 / 2) a_{i}^{2} p m_{i}+(1 / 6)\left(13 a_{i}^{2}-30 a_{i}+18\right) p m_{i-1} \\
& +27\left(3-4 a_{i}\right)^{-1}\left(f\left(y_{i}\right)-f\left(t_{i}\right)\right)+J_{i}^{-1} f\left(t_{i}\right) .
\end{aligned}
$$

Thus, from (4.3) we have

$$
\begin{aligned}
-144 a_{i+1}^{2} J_{i+1} m_{i+1}+\left(22-J_{i}^{*}\right) m_{i}-16\left(1-a_{i}\right)^{2} J_{i} m_{i-1} \\
=d^{-1}\left[-f\left(t_{i}\right)+(27 / 32) B_{i+1}(t, y)+(5 / 32) B_{i}(t, y)\right],
\end{aligned}
$$

where $J_{i}^{*}=5\left(3+4 a_{i}\right) J_{i}+27\left(5-4 a_{i+1}\right) J_{i+1}$ and

$$
B_{i}(t, y)=f\left(t_{i}\right)-27\left(3-4 a_{i}\right)^{-1} J_{i}\left(f\left(t_{i}\right)-f\left(y_{i}\right)\right) \text {. }
$$

The coefficients of $m_{i+1}$ and $m_{i-1}$ are clearly not positive since $0<a_{i}<1$. Thus, we see that the difference of the coefficient of $m_{i}$ over the sum of the positive values of the coefficients of $m_{i-1}$ and $m_{i+1}$ is

$$
8\left(4-5 J_{i}-27 J_{i+1}\right)
$$

which is positive, since for $0 \leqslant a_{i} \leqslant 4 / 5, J_{i}$ does not exceed (1/8.36). This, of course, implies that the coefficient of $m_{i}$ is positive. Thus, the coefficient matrix of the equation (4.4) is diagonally dominant and we have proved the following.

THEOREM 3. Let $f$ be 1-periodic and for all $i$ either $0<a_{i}<3 / 4$ or $3 / 4<a_{i}<$ $4 / 5$. Then there exists a unique spline $s(x)$ in the class $S^{*}(3, P)$ satisfying the conditions of Problem B.

Remark 4.1. The interpolation Problem B for equispaced points of interpolation $y_{i}=x_{i-1}+l p$ and $t_{i}=x_{i-1}+m p$ has been studied for nonequidistant knots in [3, Theorem 2] under the restriction that $1 / 2<l+m<3 / 2$. Taking $m=3 / 4$ it may be observed that Theorem 3 has the scope of covering interpolatory conditions for a wider choice of $l$ for equidistant knots. For example, if we take $a_{i}=l$ for all $i$ in Theorem 3 then for the case $3 / 4<a_{i}<4 / 5,3 / 4+l>3 / 2$.

5. Error bounds (two point interpolation). In order to estimate the error for the spline interpolant of Theorem 3, we see that the right side of the equation (4.4) is

$$
\begin{aligned}
32 p^{-1} & {\left[\left(f\left(t_{i+1}\right)-f\left(t_{i}\right)\right)-27 G_{i+1}(t, y)-5 G_{i}(t, y)\right] } \\
& =32 f^{\prime}\left(v_{i}\right)-216 J_{i+1} f^{\prime}\left(u_{i+1}\right)-40 J_{i} f^{\prime}\left(u_{i}\right)
\end{aligned}
$$

where the points $u_{i}$ are in $\left(t_{i}, y_{i}\right)$, the points $v_{i}$ are in $\left(t_{i}, t_{i+1}\right)$ and $G_{i}(t, y)=$ $J_{i}\left(3-4 a_{i}\right)^{-1}\left(f\left(t_{i}\right)-f\left(y_{i}\right)\right)$.

Thus, writing $e=s-f$ and

$$
F(z, i)=f^{\prime}\left(z_{i}\right)-f_{i-1}^{\prime}, \quad F(i, z)=f^{\prime}\left(z_{i}\right)-f_{i}^{\prime},
$$

we see that (4.4) may be written as

$$
\begin{aligned}
-144 a_{i+1}^{2} J_{i+1} e_{i+1}^{\prime}+\left(22-J_{i}^{*}\right) e_{i}^{\prime}-16\left(1-a_{i}\right)^{2} J_{i} e_{i-1}^{\prime} \\
=32 F(i, v)+9 \Delta f_{i}^{\prime}-\Delta f_{i-1}^{\prime}-27 F_{i+1}^{*} \\
-5 F_{i}^{*}-108 a_{i+1} J_{i+1} \Delta f_{i}^{\prime}-20 a_{i} J_{i} \Delta f_{i-1}^{\prime}
\end{aligned}
$$

where $F_{i}^{*}=J_{i}(5 F(u, i)+3 F(i, u))$. 
As already observed, $J_{i}$ does not exceed (1/8.36) for relevant values of $a_{i}$ and hence the expression in (4.5) is not less than 288/209. Thus the row max norm of the inverse of the coefficient matrix in (4.4) does not exceed 209/288. In view of this it follows from (5.1) that

$$
\left|e_{i}^{\prime}\right|<K\left(a_{i}\right) w\left(f^{\prime} ; p\right)
$$

where $K\left(a_{i}\right)$ is a positive function of $a_{i}$. Hence by a reasoning used already in [3, p. 249], we have

$$
\left\|e^{\prime}\right\|<K w\left(f^{\prime} ; 1 / n\right)
$$

where $K$ is some positive constant.

We have thus proved the following:

THEOREM 4. Let $f$ be a 1-periodic function in the class $C^{1}[0,1]$ and $s(x)$ be its spline interpolant in $S^{*}(3, P)$ of Theorem 3. Then for $r=0,1$,

$$
\left\|(s-f)^{(r)}(x)\right\|<(1 / n)^{1-r} K w\left(f^{\prime} ; 1 / n\right)
$$

where $K$ is a positive constant.

(5.3) is (5.4) with $r=1$. The other inequality follows directly by integration.

\section{REFERENCES}

1. C. de Boor, A practical guide to splines, Springer-Verlag, New York, 1978.

2. H. P. Dikshit, On cubic spline interpolation, J. Approximation Theory 22 (1978), 105-111.

3. A. Meir and A. Sharma, Convergence of a class of interpolatory splines, J. Approximation Theory 1 (1968), 243-250.

4. A. Sharma and J. Tzimbalario, Quadratic splines, J. Approximation Theory 19 (1977), 183-193.

Department of P. G. Studies and Research in Mathematics, University of Jabalpur, JabalpUr, 482001 INDIA 